This item was submitted to Loughborough's Research Repository by the author.

Items in Figshare are protected by copyright, with all rights reserved, unless otherwise indicated.

\title{
Gait motion analysis based on WB-4 sensor with quaternion algorithm
}

PLEASE CITE THE PUBLISHED VERSION

http://dx.doi.org/10.1109/CYBER.2016.7574836

PUBLISHER

(c) IEEE

VERSION

AM (Accepted Manuscript)

LICENCE

CC BY-NC-ND 4.0

REPOSITORY RECORD

Wang, Chunbao, Tongyang Sun, Lihong Duan, Quanquan Liu, Zhijiang Lu, Meng Li, Pengfang Chen, et al.. 2019. "Gait Motion Analysis Based on WB-4 Sensor with Quaternion Algorithm". figshare.

https://hdl.handle.net/2134/25947. 


\title{
Gait Motion Analysis Based on WB-4 Sensor with Quaternion Algorithm *
}

\author{
C.b. Wang, T.y. Sun, L.h. Duan ${ }^{1}$, Q.q. Liu, Z.j. Lu, M. Li, P.f. Chen, C.d. Wei, A.h. Hou, Y.j. Shen, Q.h. Liu, \\ J. Qin, W.g. Li, Q. Shi, Y.l. Wang, J.j. Long, J.j. Wei, M. ZECCA and Z.z. Wu
}

\begin{abstract}
Up to now, with the increasing of the elderly population, more and more patients are suffering from hemiplegia. The needs for hemiplegic rehabilitation is increasing quickly. As traditional rehabilitation, each patient must be treated by therapist, one by one. However, because of the different levels of therapists, the rehabilitation cannot be performed as the as the same. Normally, the rehabilitation status diagnosing is still be performed by therapists with the subjective experience. It caused the inhomogeneity on rehabilitation evaluation. It also sometimes causes negative influence on the rehabilitation effect. To solve these problems, many researches focusing on assessing the status of the hemiplegic patients quantitatively are proposed rehabilitation evaluation systems. Rehabilitation motion detection is the basis of the evaluation system, and it requires the participation of therapist. In this paper, a method is presented to detect lower limb motion of hemiplegic patients based on inertial sensor technology. The gesture quaternion of lower limb can be obtained through LPMS. With the matrix and Euler angle changing algorithm, combining with the simplified lower limb motion model, the rotation angle
\end{abstract}

Corresponding Author: Lihong Duan, Zhengzhi Wu work in the Shenzhen Institute of Geriatrics. Address: Shenzhen Second People's Hospital, No. 3002, Sungang Xi Road, Shenzhen, 518037, P.R China. ( mrwang@fuji.waseda.jp).

Chunbao Wang is affiliated with the First Affiliated hospital, Sun Yat-sen University and Shenzhen Institute of Geriatrics \& Shenzhen Second People's Hospital. He is also a visiting researcher of Hongkong City University and invited researcher of Waseda University.

Tongyang Sun, Zhijiang Lu, Qihong Liu and Weiguang Li are affiliated with the School of Mechanical and Automotive Engineering, South China University of Technology, China (E-mail: suntyam@163.com).

Pengfang Chen, Chengdong Wei and Anxin Hou are affiliated with MK Smart Robot Research Co.Ltd.

Yajing Shen is affiliated with the Mechanical and Biomedical Engineering, City University of Hong Kong.

Meng Li, Quanquan Liu are affiliated with Shenzhen Institute of Geriatrics and Shenzhen Second People's Hospital.

Jianjun Long, Yulong Wang is affiliated with Shenzhen Second People's Hospital.

Jianjun Wei is affiliated with Guangxi University of Science and Technology, China.

Jian Qin is a professor affiliated with the Fist Affiliated Hospital, Sun Yat-sen University.

Qing Shi is affiliated with School of Mechatronical Engineering, Beijing Institute of Technology. He is also a visiting researcher of Takanishi Lab, Waseda University.

Massimiliano ZECCA is with School of Electronic, Electrical and Systems Engineering. Sir David Davis Building, W2.61, Loughborough University, Leicestershire LE11 3TU, United Kingdom.

*Research supported by Shenzhen Honkong Joint research Project (No.SGLH20150211093607169); Postdoctoral Science Foundation of China (No. 2015M580759); Technology Research Foundation of Basic Research Project of Shenzhen ( JCYJ20150330102401102, JCYJ20150401171152771); Science and Technology Department of Guangdong(2014A020215004, 2016A020220001); Guangxi Key Scientific and Technological Project (1598013-12) of joint can be computed. Finally, the curve of rotation angle of knee is established.

\section{INTRODUCTION}

Aging problem takes many social problems up t now [1][2]. The number of hemiplegia is increasing quickly. It has been a high incidence disease and high recurrence rate among aged people. About $75 \%$ of survivors after stroke have disability, as well as $40 \%$ have severely disabled [3].

The correct diagnosis on motion disorder takes an important role to perform an effective treatment. Currently, subjective judgment is restricted by the experience of therapists. It takes a dominant position in clinical diagnosis. How to present the patient status objectively is the basic needs for developing the best treatment for matching patient status and shorting the rehabilitation period. However, there are few researches focusing on motion detection and parametric description for motion function of hemiplegic patient.

Motion detecting and parametric description are the main components of integral evaluation system. There are many motion detecting methods have been developed in past researches. Mechanical tracking system, as the most primitive motion tracking technology, possess characteristics of high tracking accuracy and fast response, such as WB-4 of Waseda University [4], SLERT of National Taiwan University [5]. It can be applied in human motion tracking, remote operation, rehabilitation medicine and virtual reality simulation. However, because of the human interval differences, the mechanical tracking system must be recalibrated for individuals. It requires more long calibration term. And more, it is difficult to capture the motion information of the patient accurately due to the bulkiness of the mechanism system.

Given the complexity of rehabilitation environment, mechanical tracking having a complex calibration, optical sensor interfered by therapists, acoustic tracking's low accuracy and electromagnetic tracking vulnerable to metal interference, many motion detection methods do not meet the detection requirements. Optical detecting, such as Vicon [6], OPTOTRAK of Northern Digital [7], STAGE of Organic Motion [8] and Kinect of Microsoft [9], plays an important role in motion tracking system. These non-contact testing methods don't restrict the normal movement of the body, thus the detected data can reflect the movement more truly. However, it would be disturbed by robot or therapist, and requires a limited detecting area.

Inertial sensing technology is a relatively new motion tracking system with high performance, easy wearable, portable and large measurement range of advantages. Takanishi Laboratory of Waseda University developed an inertial sensing system: WB sensor, which can be used to detect lower limbs with 8 IMU and 12 IMUs for the upper 
limbs. All the communication between PC and IMUs are transmitted via Bluetooth wireless devices. Therefore, the process of installation is very convenient. The system has been applied to abdominal surgery successfully [10].

\section{TOOLS}

\section{A. Simplified Human Motion Model}

Human body motion model is an abstraction of the human movement in the real word. The substance of the stick figure model is regarding the human body motion as the human bones motion, and it express various parts of human body in a straight line approximated. For example, sticks figure model proposed by Chen and Lee [11] contains 17 sections and 14 connection points to represent the head, torso and limbs. The simplified human motion model and used herein is shown in Fig.1.
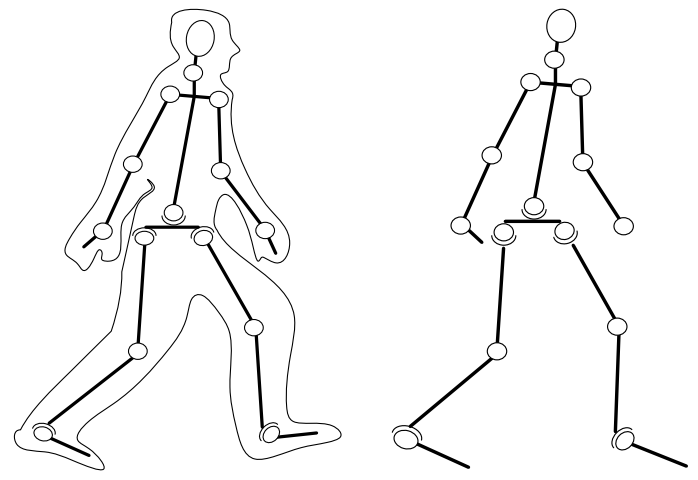

Figure 1. The Simplified Human Motion Model

For the human lower limb, the crus is the offspring of the thigh, motion information of knee is the rotation angle between crus and thigh. Human skeleton model used in this paper regards the human skeleton as rigid rod and simplifies knee to a uniaxial joint.

\section{B. Quaternion}

Hamiltion first proposed the concept of quaternion in 1843 [12]. Quaternion applied to the attitude expression and the calculation can avoid singular phenomenon brought by Euler angles.

\section{1) the Unit Norm Quaternion:}

A special subset of the quaternion space, denoted $\mathbf{I H}$, is defined when $\|\mathrm{q}\|=1$, then $\mathrm{q}$ is called an unit norm quaternion and the unit quaternion space is denoted $\mathbf{I H}_{1}$. This particular subset is of special interest since it allows the characterization of orientation trajectories. The temporal orientation trajectories are studied in the unit norm quaternion space $\mathbf{I H}_{1} \subset \mathbf{I H}$ [13]. Any general three-dimensional rotation can be transform into an unit norm quaternion $\mathrm{q} \in \mathbf{I H}_{1}$.

\section{2) the Rotation Quaternion:}

In this paper, the rotation quaternion can be expressed with $\mathrm{q}=(w, x, y, z)^{T}$, where $w, x, y, z$ are real numbers, and $w$ is the cosine of half of rotation angle, and $x, y, z$ are the multiplication $\mathrm{X}, \mathrm{Y}, \mathrm{Z}$ coordinates of rotation axis and the sine of half of rotation angle, and $w^{2}+x^{2}+y^{2}+z^{2}=1$.

\section{Life Performance Research Motion Sensor (LPMS)}

Inertial sensing technology is a relatively new motion tracking system with high performance, easy wearable, portable and large measurement range of advantages. The Life Performance Research Motion Sensor (LPMS) is selected as the motion sensor. The LP-Research Motion Sensor Bluetooth version (LPMS-B) is a miniature wireless inertial measurement unit (IMU) / attitude and heading reference system (AHRS). The unit is very versatile, performing accurate, high speed orientation and displacement measurements. By the use of three different MEMS sensors (3-axis gyroscope, 3-axis accelerometer and 3-axis magnetometer) drift-free, high-speed orientation data around all three axes is achieved. The LPMS-B communicates with a host system via a Bluetooth connection. The LPMS sensor calculates the orientation difference between a fixed sensor coordinate system and a global reference coordinate system.

$$
\mathrm{Q}_{\text {Sensor }}=q_{\text {Difference }} Q_{\text {Global }} q_{\text {Difference }}{ }^{-1}
$$

where $\mathrm{Q}_{\text {Sensor }}$ is the fixed sensor coordinate system, and $Q_{\text {Global }}$ is the global coordinate system, $q_{\text {Difference }}$ is the orientation difference between $Q_{\text {Global }}$ and $\mathrm{Q}_{\text {Sensor }}$.

The local and the global reference coordinate systems used are defined as right handed Cartesian coordinate systems with: $\mathrm{X}$ positive when pointing to the magnetic west, $\mathrm{Y}$ positive when pointing to the magnetic south, and $\mathrm{Z}$ positive when pointing up (gravity points vertically down with -1 g). The axis orientation of LPMS-B and the relationship between local sensor coordinate system and global coordinates are shown as Fig.2.

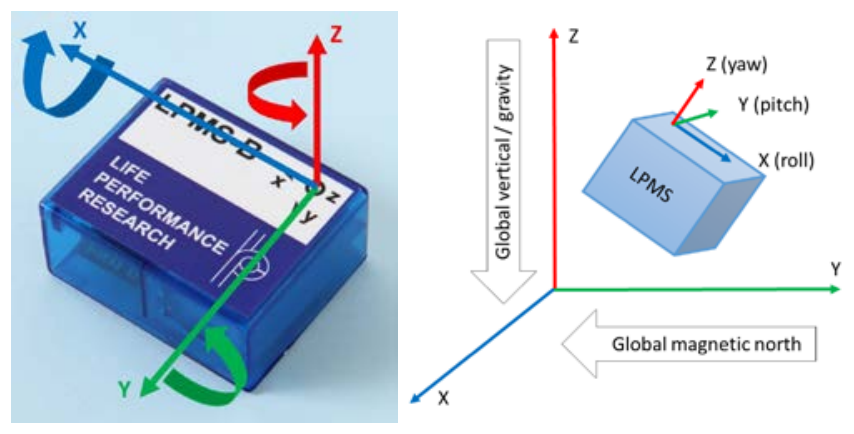

Figure 2. Relationship Between Different Coordinates

\section{METHODS}

Some principles of the two-link skeletal model should be addressed first before getting the rotation angle of human lower limb. The two-link skeletal model is proposed to connect the basic grid body with inertial sensor and express the gesture of grid body in angle.

\section{A. Two-Link Skeletal Model}

The motion of the knee can be simplified to a single degree of freedom articulation as the simplified motion model discussed above. Motion sensors mounted on the body of the thigh and crus can be simplified a rotation around a single axis. An experiment is designed to verify the correctness of the algorithm about the rotation around the single articulation by using the plank experiment to simulate the knee joint motion. 
Two sensors are fixed on two different planks and the coordinate system is set as shown in Fig.3. The first sensor is indicated as S1 and the second as S2, a stick is fixed on the center of two planks.

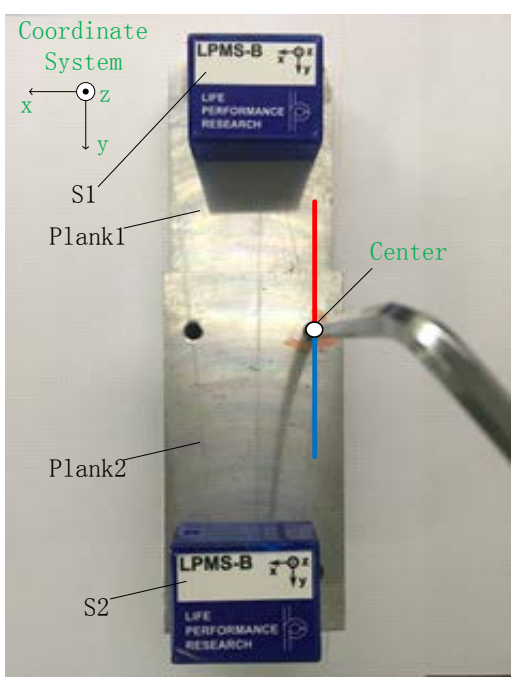

Figure 3. Sensors Mounting Position

The sensor motion schematic is shown in Fig.4, where S1 keeps its position and S2 anticlockwise rotates 90 degree from its initial position. Next, plank2 is rotated to its original position, and a set of data is recorded.

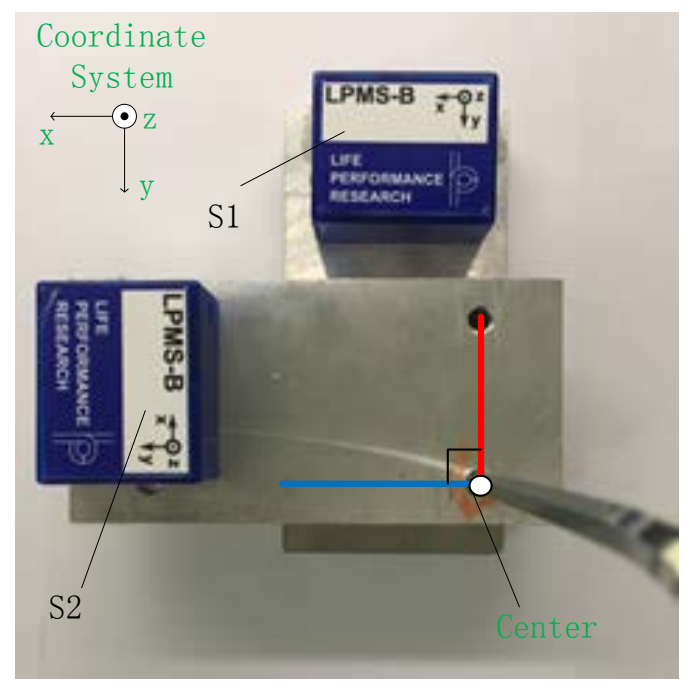

Figure 4. Sensors Motion Schematic

$\mathrm{Q}_{1}$ and $\mathrm{Q}_{2}$ are defined to express the quaternion of $\mathrm{S} 1$ and S2, using 1 corresponding to sensor- 1 and 2 corresponding to sensor-2. The attitude of two sensors at the initial moment $T_{0}$ are $Q_{01}$ and $Q_{02}$, and the rotation quaternion are $q_{01}$ and $q_{02}$,

$$
\begin{aligned}
& \mathrm{Q}_{01}=\mathrm{q}_{01} \mathrm{Q}_{0} \mathrm{q}_{01}{ }^{-1} \\
& \mathrm{Q}_{02}=\mathrm{q}_{02} \mathrm{Q}_{0} \mathrm{q}_{02}{ }^{-1}
\end{aligned}
$$

where $Q_{0}$ is represented the same global reference coordinate system of two sensors, and the equation expressing $\mathrm{Q}_{0}$ by $\mathrm{Q}_{01}$ can be obtained as follows,

$$
\mathrm{Q}_{0}=\mathrm{q}_{01}{ }^{-1} \mathrm{Q}_{01} \mathrm{q}_{01}
$$

And then, another equation from equation (3) and equation (4) can be expressed as follows,

$$
\mathrm{Q}_{02}=\mathrm{q}_{02} \mathrm{q}_{01}{ }^{-1} \mathrm{Q}_{01} \mathrm{q}_{01} \mathrm{q}_{02}{ }^{-1}=\mathrm{q}_{0} \mathrm{Q}_{01} \mathrm{q}_{0}{ }^{-1}
$$

where the rotation $q_{0}=q_{02} q_{01}^{-1}$ is used to correct the data between sensor-1and sensor- 2 at the initial moment $\mathrm{T}_{0}$.

The attitude of two sensors are defined with $Q_{11}$ and $Q_{12}$ at any moment $T_{1}$ in motion, and the rotation quaternion $q_{11}$ and $\mathrm{q}_{12}$,

$$
\begin{aligned}
& \mathrm{Q}_{11}=\mathrm{q}_{11} \mathrm{Q}_{0} \mathrm{q}_{11}{ }^{-1} \\
& \mathrm{Q}_{12}=\mathrm{q}_{12} \mathrm{Q}_{0} \mathrm{q}_{12}{ }^{-1}
\end{aligned}
$$
(4),

Another equation is derived from equation (6) as equation

$$
\mathrm{Q}_{0}=\mathrm{q}_{11}{ }^{-1} \mathrm{Q}_{11} \mathrm{q}_{11}
$$

Next, using the data at $\mathrm{T}_{0}$ to correct the data of sensors, and

$$
\mathrm{Q}_{11}{ }^{\prime}=\mathrm{q}_{0} \mathrm{Q}_{11} \mathrm{q}_{0}{ }^{-1}
$$

And, another equation is computed as follows,

$$
\mathrm{Q}_{11}=\mathrm{q}_{0}{ }^{-1} \mathrm{Q}_{11}{ }^{\prime} \mathrm{q}_{0}
$$

A new equation computed by equation (5), (7), (8), (10) can be expressed the correction as follows,

$$
\begin{aligned}
\mathrm{Q}_{12} & =\mathrm{q}_{12} \mathrm{Q}_{0} \mathrm{q}_{12}{ }^{-1}=\mathrm{q}_{12} \mathrm{q}_{11}{ }^{-1} \mathrm{Q}_{11} \mathrm{q}_{11} \mathrm{q}_{12}{ }^{-1} \\
& =\mathrm{q}_{12} \mathrm{q}_{11}{ }^{-1} \mathrm{q}_{0}{ }^{-1} \mathrm{Q}_{11}{ }^{\prime} \mathrm{q}_{0} \mathrm{q}_{11} \mathrm{q}_{12}{ }^{-1}=\mathrm{q}_{1} \mathrm{Q}_{11} \mathrm{q}_{1}{ }^{-1}
\end{aligned}
$$

where

$$
\mathrm{q}_{1}=\mathrm{q}_{12} \mathrm{q}_{11}^{-1} \mathrm{q}_{0}^{-1}=\mathrm{q}_{12} \mathrm{q}_{11}^{-1} \mathrm{q}_{02} \mathrm{q}_{01}{ }^{-1}
$$

is represented the correction rotation quaternion between S1 and S2.

The conversion of quaternion to Euler angles is used to get the rotation angle. The definition used for Euler angles in this paper is equivalent to roll, pitch, yaw. The Euler angles are of ZYX global type (subsequent rotation around global Z, Y and $\mathrm{X}$ axis, also known as aerospace sequence).

Convert the rotation quaternion $\mathrm{q}=(w, x, y, z)^{T}$ to matrix, the rotation matrix $\mathrm{M}$ as follows,

$\mathrm{M}=$

$\left[\begin{array}{ccc}w^{2}+x^{2}-y^{2}-z^{2} & 2(x y-z w) & 2(x z+y w) \\ 2(x y+z w) & w^{2}-x^{2}+y^{2}-z^{2} & 2(y z-x w) \\ 2(x z-y w) & 2(y z+x w) & w^{2}-x^{2}-y^{2}+z^{2}\end{array}\right]$

And the conversion of the rotation matrix $\mathrm{M}$ to Euler angles is shown as follows,

$$
\begin{gathered}
\text { EulerY }=\operatorname{atan} 2\left(\mathrm{M}_{13}, \sqrt{{M_{11}}^{2}+{M_{12}}^{2}}\right) \\
\text { EulerZ }=\operatorname{atan} 2\left(-\frac{\mathrm{M}_{12}}{\cos (\text { EulerZ })}, \frac{\mathrm{M}_{11}}{\cos (\text { EulerZ })}\right) \\
\text { EulerX }=\operatorname{atan} 2\left(-\frac{\mathrm{M}_{23}}{\cos (\text { EulerZ })}, \frac{\mathrm{M}_{33}}{\cos (\text { EulerZ })}\right)
\end{gathered}
$$

where the element of i-th row and j-th column of matrix $\mathrm{M}$ can be represented as $\mathrm{M}_{i j}$. 
The curve of rotation angle can be computed with Matlab by processing the data collected by the sensor as shown in Fig.5.

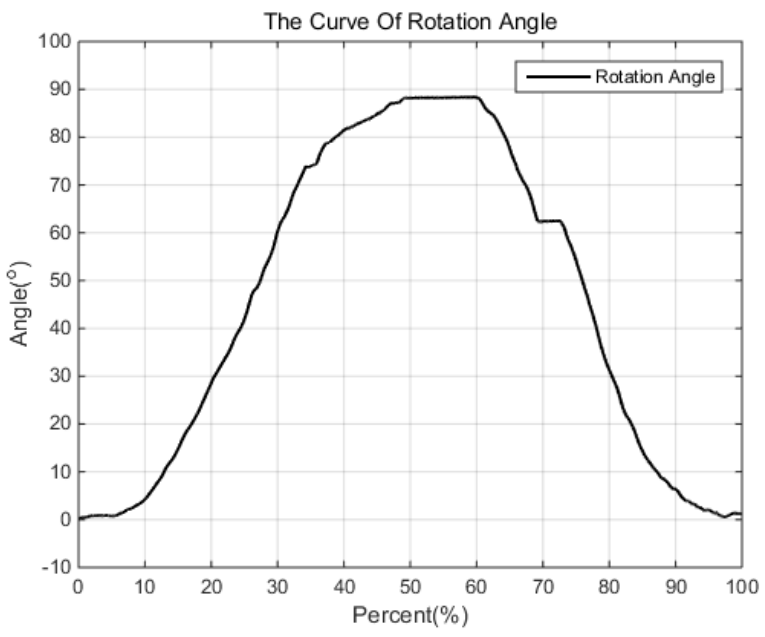

Figure 5. The Curve Of Angle Between S1 And S2

The rotation angle of two sensors around the center of S1 and S2 is shown in Fig.5. There is a little error $\left(2^{\circ}\right)$ in the highest point, and it is possible caused by the rotation operation. And the result of the two-link skeletal model experiment meets the experiment expectations and verifies the correctness of the algorithm.

\section{EXPERIMENTS AND RESULTS}

A series of experiments has been established to get the curve of human lower limb motion.
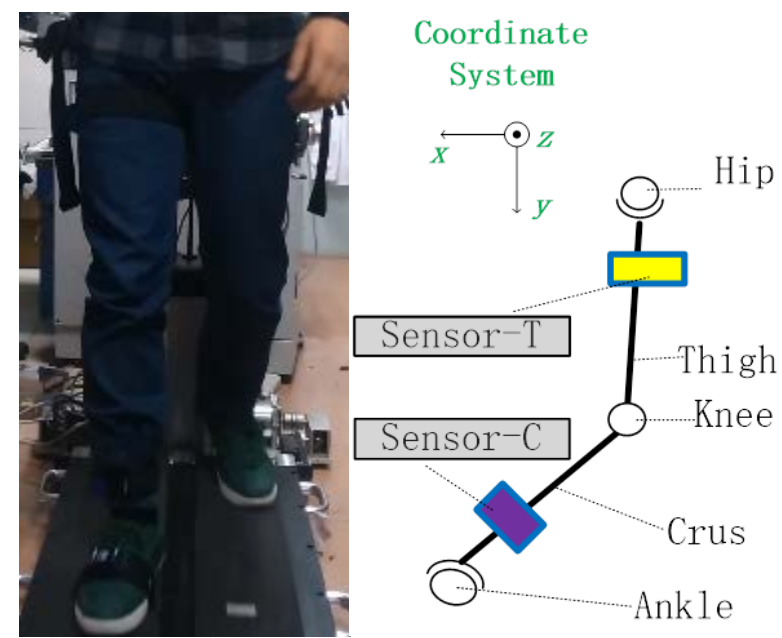

Figure 6. The Gait Motion Analysis Experiment

Sensors are attached on thigh and crus in the experiment as shown in Fig.7. Sensor-T stands for the sensor of thigh and sensor-C for sensor of crus. A set of data is recorded in the experiment when lower limb is in motion and three experiments have been conducted. In each experiment, human starts moving from stationary.

The curve of knee rotation angle can be computed with Matlab by processing the data collected by the sensor as shown in Fig.8.
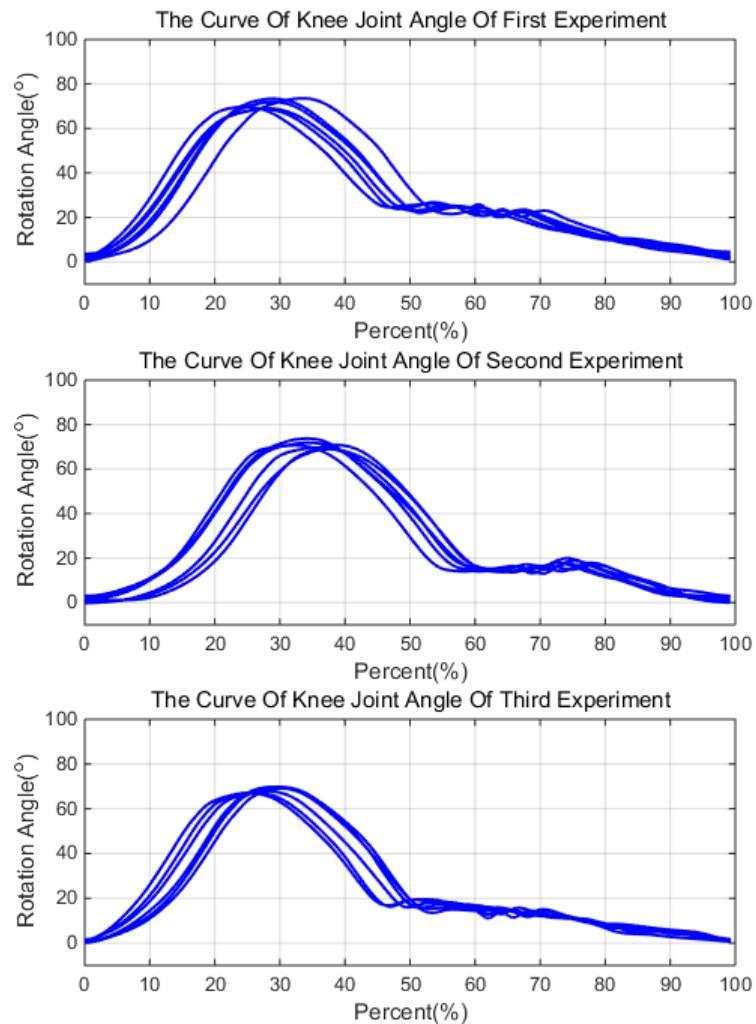

Figure 7. The Curve Of Knee Joint Angle

As shown from the curve of knee joint angle in Fig.8, the rotation angles of human knee joint changes periodically in motion. In each cycle, the curve of angle has a significant feature, the rotation angle increases rapidly and then decreases to the vicinity of a smaller value, and then decreases slowly. However on human lower limb model experiment, the muscle elasticity and body vibration will slightly influence the angle output of the knee joint when human is in motion.

The data of different cycles on three experiments is shown as follows in Fig.9.

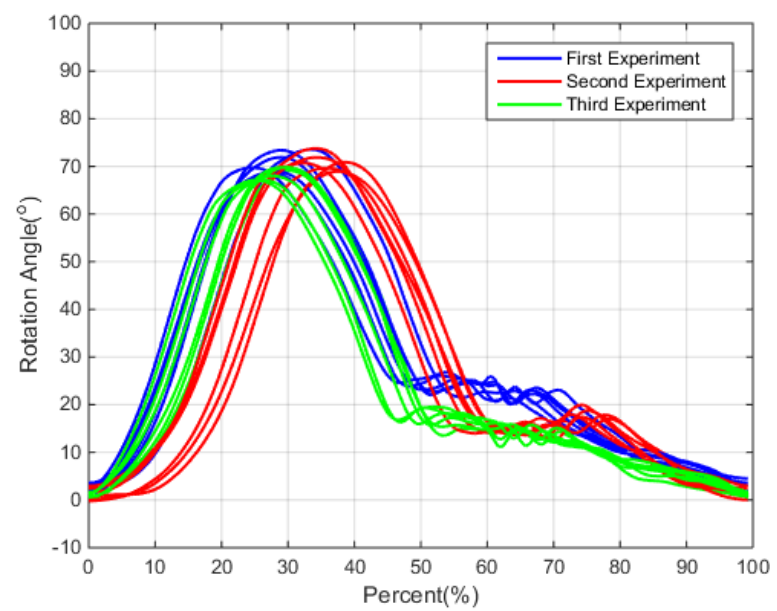

Figure 8. Rotation Angle Of Knee 
From the experiment conducted above, this novel lower limb motion detecting method based on LPMS is practical and reliable. The gesture quaternion of lower limb based on LPMS can be converted to matrix and Euler angle. The rotation angle of joint can be computed by combined with the simplified lower limb motion model. Finally, the curve of rotation angle of knee is established by using the algorithm.

\section{CONCLUSION AND FUTURE WORK}

Gait motion detection takes an important role in the patient evaluation. In this paper, a novel method to detect lower limb motion based on inertial sensor technology is proposed. The gesture quaternion of lower limb can be obtained through LPMS with the algorithm of converting quaternion to matrix and Euler angle. The simplified lower limb motion model is also proposed in the paper. Combining with the algorithm and model, the rotation angle of joint is calculated out. With Matlab the rotation quaternion is processed and the curve of rotation angle of knee is established. All the curves show the possibility to analysis the human gait motion with the proposed method.

As a future work, the correction of experiment model and calibration of motion error are needed to be perfected to achieve better functionality. Different types of experiments also need to be done. The method detecting the motion of lower limb can be integrated into the rehabilitation robot control system, realizing intelligent detection and evaluation. Thus, the rehabilitation robots could be expected adjusting training parameters based on patient status automatically. Once the improvements have been incorporated into the system, experiments will be arranged among hemiplegic patients to confirm the effectiveness of motion detecting system, rehabilitation robot control system and rehabilitation evaluation system. This research will have significant influence on motion detection, rehabilitation evaluation and medical rehabilitation robot fields.

\section{REFERENCES}

[1] Aging population development trend forecasting research reports of China. Members of the National Council on Aging office .2006.

[2] State Department China Aging Development "Twelve Five" plan, 2011.9.17: 2.

[3] Jishu Rong. Practical hemiplegia rehabilitation technical illustration. People's Medical Publishing House .2005

[4] Sarah Cosentino; Klaus Petersen; Zhuohua Lin; Luca Bartolomeo; Salvatore Sessa; Massimiliano Zecca; Atsuo Takanishi.Natural human-robot musical interaction: understanding the music conductor gestures by using the WB-4 inertial measurement system[J] Advanced Robotics, 2014,28(11):781-792.

[5] Wu T. M., Chen D. Z. Biomechanical study of upper-limb exoskeleton for resistance training with three-dimensional motion analysis system [J]. Journal of Rehabilitation Research \& Development. 2014, 51(1):111-126.

[6] Duffell L.D., Hope N., McGregor A.H. Comparison of kinematic and kinetic parameters calculated using a clusterbased model and Vicon's plug-in gait [J]. Proceedings of the Institution of Mechanical Engineers, Part H: Journal of Engineering in Medicine, 2014, 228(2):206-210.
[7] Zhou J., Yu Y.Q. Coordination control of dual-arm modular robot based on position feedback using Optotrak3020 [J]. Industrial Robot, 2011 , 38(2):172-185

[8] Organic Motion Inc.,[Online]. Available:http://www.Organicmotion. com.

[9] Pfister A., West A.M., Bronner S., Noah J.A. Comparative abilities of Microsoft Kinect and Vicon 3D motion capture for gait analysis [J]. Journal of Medical Engineering and Technology, 2014, 38(5):274-280.

[10] J Zhuohua Lin; Munenori Uemura; Massimiliano Zecca; Salvatore Sessa; Hiroyuki Ishii; Morimasa Tomikawa; Makoto Hashizume; Atsuo Takanishi. Objective skill evaluation for laparoscopic training based on motion analysis [J].Advanced Robotics, 2013;60(4):977-985.

[11] Z.Chen,H.J.Lee. Knowledge-guided visual perception of 3D human gait from a single image sequence. IEEE Transactions on Systems Man CYberneticsl [J], 1992,22(2):336-342

[12] Dezhong Lian. The Real Transformations of Quaternion Vector and Matrix [J]. Journal of XiaMen University, 2003. 42(6).

[13] J. B. Kuipers. Quaternions and rotations sequences: A primer with applications to orbits, Aerospace, and virtual reality [J]. Princeton University Press, 1999. ISBN 0-691-05872-5. 